\title{
Autoimmunphänomene unter Checkpointinhibitoren
}

Immuntherapien haben zu einem Paradigmenwechsel in der Onkologie geführt - und bescheren auch den Rheumatologen ein neues Aufgabengebiet, kommt es doch unter Therapie mit den inzwischen breit angewendeten Immuncheckpointinhibitoren häufig zu Autoimmunphänomenen.

"Etwa $30-40 \%$ unserer Patienten in der onkologischen Praxis bekommen zu einem Zeitpunkt ihrer Therapie [...] einen Immun-Checkpointinhibitor", so Prof. Dr. Heinz Läubli. Er ist leitender Oberarzt der Onkologie am Universitätsspital Basel und leitet dort das Immuntherapielabor. Beispiele für Checkpointinhibitoren (CPi) sind der gegen den T-ZellOberflächenrezeptor CTLA4 gerichtete Antikörper Ipilimumab oder der PD1-Inhibitor Nivolumab, der unter anderem beim malignen Melanom und kleinzelligen Bronchialkarzinom eingesetzt wird.

》) Anders als Chemotherapien wirken Checkpointinhibitoren nicht direkt am Tumor

Anders als Chemotherapien wirken Checkpointinhibitoren (CPi) nicht direkt am Tumor, sondern indirekt, indem sie an Rezeptoren auf Immunzellen binden. „Ein Checkpoint ist ein Regulator einer Immunaktivierung", erläuterte DGRh-Präsident Professor Hendrik Schulze-Koops aus München, der unter anderem über T-Zellen in der Pathogenese von Autoimmunerkrankungen forscht. Checkpoints (CP) seien Oberflächenmoleküle, die Veränderungen der Zellumgebung registierten und in der Zelle eine aktivierende oder blockierende Wirkung hervorriefen.

Beispiele aus der Rheumatologie sind TNF-Rezeptoren oder ko-stimulatorische Moleküle wie CD28. Es gibt pro- und anti-inflammatorische CP. Zu letzteren zählen CTLA4 und PD1, sie dämpfen die T-Zell-Aktivität. Werden sie durch CPi blockiert, löst das die
Bremsen im Immunsystem, und die Immunzellen können Tumorzellen angreifen. Doch die ungebremste Immunantwort hat ihre Tücken.

\section{Auch autoimmune T-Zellen werden aktiv}

Bei Autoimmunerkrankungen beobachtet Schulze-Koops schon länger mögliche Negativfolgen einer T-Zellaktivierung. Er verwies auf das Umdenken im Verständnis von Autoimmunität: "Die Existenz von Autoimmunzellen ist völlig physiologisch." - Nicht so deren Aktivierung. Sie passiere "in dem Moment, wo man die klassischen Regulationsmechanismen CTLA4 und PD1/PDL1 blockiert." Rheumatherapien zielen auf eine Herunterregulierung der Immunaktivierung. Wenn „wir die T-Zell-Aktivierung blockieren, während die Onkologen jetzt die T-Zell-Deaktivierung blockieren", sei nachvollziehbar, dass CPi auch autoimmune T-Lymphozyten überaktivierten, „die letztlich zur Zerstörung nicht nur des Tumors, sondern auch gesunden Gewebes führen." Jedes Organ, könne angegriffen werden.

Etwa $70 \%$ der Patienten entwickelten immune-related adverse events (irAE), meist 3-6 Monate nach
Therapiebeginn. Er habe aber auch eine Patientin mit foudroyanter Polyarthritis am ersten Tag nach Therapiebeginn gesehen, ebenso ungewöhnliche Autoimmunreaktionen mehrere Jahre nach CPi-Therapie.

In onkologischen Beobachtungsstudien zu rheumatischen irAE - bei denen Schulze-Koops einen starken „Bias zum Underreporting" vermutet - wurden Arthralgien bei bis zu $43 \%$, Arthritiden bei bis zu $7 \%$, Myalgien bei bis zu $21 \%$, und ein Sicca-Syndrom bei bis zu 24\% der Patienten beschrieben [1].

Einzelfälle ähnelten Vaskulitiden, SLE, Polymyalgia rheumatica oder Sarkoidose, man sehe Aspekte von Spondyloarthritiden oder eosinophiler Fasziitis. Auch Antikörper werden nachgewiesen: CCP-Ak in bis zu $20 \%$ und ANA, meist niedrigtitrig, in bis zu $60 \%$.

Das sei nur ein unspezifischer Ausdruck immunologischer Aktivität. Er dürfe Rheumatologen nicht fehlleiten, eine klassische rheumatische Erkrankung zu vermuten: „Es sind keine Autoimmunerkrankungen, die dadurch entstehen, sondern Autoimmunphänomene." Bester Beleg dafür sei die im Gegensatz zu vielen Rheumaerkrankungen ausgewogene Geschlechterverteilung.

\section{Das Wichtigste in Kürze}

Welche Bedeutung haben autoimmune Nebenwirkungen (irAE) bei der Therapie mit Immuncheckpointinhibitoren?

Sie sind sehr häufig und mit einem guten Ansprechen auf die Immuntherapie assoziiert.

Bedeutung: Es obliegt dem Rheumatologen, die rheumatologischen irAE bei fortgesetzter CP-Therapie unter Kontrolle zu bringen.

Einschränkung: Nur bei ihrerseits lebensbedrohlichen irAE, wie schweren Myositiden sollten CPi abgesetzt werden. 


\section{Rheumatologen assistieren Onkologen}

Für die Tumorerkrankung seien irAE wohl ein positives Signal. So hätten $65 \%$ von 551 Patienten mit kompletter oder partieller Remission unter CPi irAE gehabt, aber nur $24 \%$ mit Tumorprogress [2]. Studien zeigten einen „dramatischen, signifikanten Unterschied" im progressionsfreien Überleben für Patienten mit irAE $[2,3]$.

》) Als Indikation, die Immuntherapie doch zu beenden, nennt die EULAR speziell schwere Myositiden

So sieht Schulze-Koops die Rheumatologen auch in der Pflicht, den Onkologen zu assistieren und die Autoimmunphänomene „in den Griff zu kriegen" - ohne die überlebenswichtige onkologische Therapie in Frage zu stellen. Als Mitherausgeber der EULAR-Empfehlungen zu Diagnose und Management von irAE unter CPi [4] schlägt er nach NSAR zunächst lokale, wenn nötig systemische Glukokortikoide, möglichst $<10 \mathrm{mg} / \mathrm{d}$ Prednison vor, anschließend csDMARDS wie MTX, Ciclosporin A oder Leflunomid.

"Bei Methotrexat haben wir mittlerweile keine Sorge in den Dosierungen, die wir in der Rheumatologie einsetzen", es gebe keine Kontraindikationen für die CPi. Falls erforderlich, könnten Biologika verabreicht werden: Abatacept, das selbst an CTLA angreift, würde er "eher ganz am Ende" überlegen, aber TNFalpha- oder IL-6-Inhibitoren hätten möglicherweise sogar einen positiven Effekt auf die Tumortherapie. Als Indikation, die Immuntherapie doch zu beenden, nennen die EULAR-Empfehlungen speziell schwere Myositiden. Schulze-Koops: „Es ist die lebensbedrohliche Erkrankung, die behandelt werden muss."

\section{Literatur}

1. Cappelli LC et al (2017) Immune-related adverse effects of cancer immunotherapy implications for rheumatology. Rheum Dis Clin North Am 43(1):65-78
2. Kostine M et al (2018) OP0088 Immunerelated adverse events of cancer immunotherapy - when inflammatory side effects are associated with survival: a single-centre prospective cohort study. Ann Rheum Dis 77(Suppl. 2):94-95

3. Tison A et al (2019) Safety and efficacy of immune checkpoint inhibitors in patients with cancer and preexisting autoimmune diseases: a nationwide multicenter retrospective study, Ann Rheum Dis 71(12):2100-2111

4. Kostine $\mathrm{M}$ et al (2020) EULAR points to consider for the diagnosis and management of rheumatic immune-related adverse events due to cancer immunotherapy with checkpoint inhibitors. Ann Rheum Dis Published Online First: 23 April 2020. https://doi.org/ 10.1136/annrheumdis-2020-217139

Hinweis des Verlags. Der Verlag bleibt in Hinblick auf geografische Zuordnungen und Gebietsbezeichnungen in veröffentlichten Karten und Institutsadressen neutral.

rheuma plus $2021 \cdot 20: 66-67$ https://doi.org/10.1007/s12688-02100402-5

(C) Springer-Verlag GmbH Austria, ein Teil von Springer Nature 2021
Quelle: Dr.

Bianca Bach,

SpringerMedizin.de (basierend auf der Session CheckpointInhibitoren. 48. Kongress der DGRh, München (virtuell), 9.-12.09.2020)

\section{Rot um die Augen nach Corona-Infektion: ein Alarmsignal?}

Eine Multisystembeteiligung im Sinne eines MIS-C gilt als äußerst seltene Komplikation einer SARS-CoV-2-Infektion beim Kind. Daran denken sollte man zum Beispiel, wenn der kleine Patient neben unklarem Fieber bestimmte Symptome rund um die Augen präsentiert.

Seit Frühjahr 2020 wird immer wieder von ungewöhnlichen Verlaufsformen einer SARS-CoV-2-Infektion bei Kindern berichtet, die mit deutlich erhöhten Entzündungsmarkern und einer Multisystembeteiligung, ähnlich dem Kawasaki-Syndrom (KS), einhergehen. Dieses von der CDC (Centers for Disease Control and Prevention) mittlerweile als Multisystem Inflammatory Syndrome in Children (MIS-C) bezeichnete Krankheitsbild ist mit einer Inzidenz von etwa 2 von 100.000 SARS-CoV-2-Infizierten unter 21 Jahren äußertselten; aufgrund des oft schweren Verlaufs ist jedoch eine möglichst frühzeitige Diagnostik und eventuell auch die Abgrenzung vom KS entscheidend.

》) MIS-C geht in vielen Fällen mit mukokutanen Zeichen einher

Helfen könnte dabei die Beobachtung, dass das MIS-C in vielen Fällen (Stu- dien zufolge in bis zu $74 \%$ ) mit mukokutanen Zeichen einhergeht. Diese zu charakterisieren, hat sich ein Team der New York University Grossman School of Medicine vorgenommen [1].

\section{Befunde an Haut und Schleim-} häuten bei über $80 \%$

In der retrospektiven Fallserie von Trevor K. Young und Kollegen lag die Inzidenz mukokutaner Befunde bei den insgesamt 35 Kindern mit bestä- 